\title{
Aerosol Use in the Pulmonary Function Lab
}

\author{
Gregg L Ruppel MEd RRT RPFT FAARC
}

\author{
Aerosols in the Pulmonary Function Testing Lab \\ How Aerosols Are Administered in the PFT Lab \\ Bronchodilators \\ Bronchial Challenge Agents \\ Bronchodilator Testing \\ Inhaled Bronchial Challenge Agents \\ Safety During Bronchoprovocation \\ Summary
}

\begin{abstract}
Aerosolized medications are frequently used in the pulmonary function laboratory. The $\mathbf{2}$ most common implementations are bronchodilators and bronchial challenge agents. Bronchodilator administration is not well standardized, largely because of the various methods of delivery available for clinical practice. Metered-dose inhalers used with spacer devices are the most common route for bronchodilator administration, but many laboratories use small-volume nebulizers. Interpretation of pre- and post-bronchodilator studies is confounded by the definitions of airway obstruction and bronchodilator responsiveness. Protocols for administering bronchial challenge aerosols (methacholine, mannitol, hypertonic saline) are well defined but are susceptible to some of the same problems that limit comparison of bronchodilator techniques. Bronchial challenges with inhaled aerosols are influenced not only by the delivery device but by the patient's breathing pattern, particularly in protocols that include deep inspiratory efforts. Key words: aerosols; bronchodilators; bronchial challenge; methacholine; mannitol; FEV ${ }_{l}$. [Respir Care 2015;60(6):931-940. (c) 2015 Daedalus Enterprises]
\end{abstract}

\section{Aerosols in the Pulmonary Function Testing Lab}

Aerosolized agents are an essential component of pulmonary function testing (PFT). ${ }^{1}$ Inhaled bronchodilators are widely used to assess reversibility of airway obstruction, particularly in patients suspected of having asthma. ${ }^{2}$ Measure-

Mr Ruppel is affiliated with the Division of Pulmonary, Critical Care and Sleep Medicine, St. Louis University School of Medicine, St. Louis, Missouri.

Mr Ruppel presented a version of this paper at the 53rd RESPIRATORY CARE Journal Conference, "Aerosol Drug Delivery in Respiratory Care," held June 6 and 7, 2014, in St Petersburg, Florida.

Mr Ruppel has disclosed no conflicts of interest.

Correspondence: Gregg L Ruppel MEd RRT RPFT FAARC. E-mail: ruppelg1@slu.edu.

DOI: $10.4187 /$ respcare. 03493 ment of the $\mathrm{FEV}_{1} / \mathrm{FVC}$ ratio after administration of a $\beta$ agonist is the de facto test to establish the diagnosis of COPD. ${ }^{3}$ Similarly, measurement of $\mathrm{FEV}_{1}$ following bronchodilator administration is used to categorize the severity of airway obstruction. Improvement in air flow is the primary end point in the evaluation of new inhaled bronchodilators, and PFT provides the necessary tools for assessment. The other primary use of aerosols in the PFT lab involves inhaled bronchial challenge agents. As is the case for bronchodilators, bronchial provocation using an aerosolized agent is a key tool for making or excluding the diagnosis of asthma. ${ }^{4}$ Inhalation of challenge aerosols also provides a means to quantify the degree of hyper-responsiveness.

How Aerosols Are Administered in the PFT Lab

\section{Bronchodilators}

Bronchodilators can be administered by various methods for diagnostic purposes. Typically, a $\beta$ agonist or an- 
ticholinergic drug is delivered using the same method as might be employed by the patient. These methods include metered-dose inhalers (MDIs), powder inhalers, or smallvolume nebulizers powered by compressed gas. ${ }^{5}$ In some instances, the patient may be tested using the same device and dose prescribed for him/her.

\section{Bronchial Challenge Agents}

Bronchial challenge agents typically require a specific protocol depending on the agent. ${ }^{6,7}$ Methacholine and histamine are usually administered via a small-volume nebulizer. To quantify the number of breaths of the challenge agent, a dosimeter may be used. Dosimeters can be triggered manually by the pulmonary function technologist or automatically by sensing the patient's inspiratory effort. Mannitol utilizes a proprietary powder inhaler to deliver the drug. Ultrasonic or thin-film nebulizers are used to administer hypertonic saline as an airway challenge agent.

\section{Bronchodilator Testing}

Despite the fact that inhaled bronchodilators are commonly used in conjunction with PFT, how they are administered is not universally standardized. The 2005 guidelines of the American Thoracic Society/European Respiratory Society (ATS/ERS) proposed some recommendations for bronchodilator administration. ${ }^{1}$

If the goal of measuring air flow following bronchodilator administration is to determine whether improvement is possible, patients typically do not need to withhold their medications. In fact, their treatment should be optimized and their condition stable. If the question to be answered is whether air flow limitation is reversible, then patients should withhold medications before baseline testing. Shortacting $\beta$ agonists and anticholinergics such as ipratropium should be withheld at least $4 \mathrm{~h}$ before testing. Long-acting $\beta$ agonists such as salmeterol, as well as slow-release preparations and oral aminophyllines, should be withheld for at least $12 \mathrm{~h}$.

The ATS/ERS 2005 spirometry guidelines ${ }^{1}$ suggest the following steps to standardize bronchodilator administration in the context of PFT: 1) use a valved holding chamber; 2) inhale $90 \mu \mathrm{g}$ (90 $\mu \mathrm{g}$ in the United States equals $100 \mu \mathrm{g}$ in Europe because of how the dose is measured) of short-acting $\beta$ agonist (albuterol or similar) from below functional residual capacity to total lung capacity; 3 ) breathhold for 5-10 s; 4) take 4 separate doses (total of $360 \mu \mathrm{g}$ ) at 30-s intervals, and 5) repeat spirometry after 10-15 min for short-acting $\beta$ agonists and $30 \mathrm{~min}$ for short-acting anticholinergic agents.

This protocol assumes the use of an MDI delivers $\sim 360 \mu \mathrm{g}$ of drug to the airways. Other drugs may be used and at lower doses if clinically indicated. If an anticholin- ergic such as ipratropium is used, 4 puffs of $40 \mu \mathrm{g}$ may be administered. The choice of whether to use a $\beta$ agonist or an anticholinergic for reversibility testing is usually made based on the anticipated or prescribed therapy for an individual patient. For short-acting $\beta$ agonists, spirometry is performed after waiting at least $10 \mathrm{~min}$; for anticholinergics, a 30-min delay (or slightly longer) is recommended. If the bronchodilator is delivered via small-volume nebulizer, a standard dose (such as $2.5 \mathrm{mg}$ of albuterol in $2.5 \mathrm{~mL}$ of solution) should be adopted, with waiting times similar to those used for an MDI after the solution has been completely nebulized. Small-volume nebulizers with aerosol outputs in the respirable range (1-5- $\mu \mathrm{m}$ mass median aerodynamic diameter) capable of delivering the charge dose in a reasonable interval (5-15 $\mathrm{min}$ ) should be used.

The amount of drug delivered to the airways depends on the method of administration along with the particle size distribution, inspiratory flow, breath-hold time, and inspired volume. ${ }^{8}$ Each laboratory should control the method used to standardize the definition of reversibility. The use of a valved holding chamber or similar device is recommended. ${ }^{9}$ For both adult and pediatric patients, activation of the MDI by the technologist (rather than the patient) along with appropriate coaching of the inspiratory maneuver may achieve more consistent drug delivery. PFT labs should select appropriate devices (ie, MDIs, powder inhalers, small-volume nebulizers) based primarily on their particle deposition characteristics. ${ }^{10}$ For pediatric patients, a well-fitting mask rather than a mouthpiece may be needed for optimal drug delivery.

Infection control of aerosol devices used in the PFT lab closely follows the same recommendations for devices used therapeutically. ${ }^{11}$ Technologists should wear gloves while handling mouthpieces, masks, or other components that may come in direct physical contact with mucous membranes. Reusable nebulizers, mouthpieces, and other equipment must be cleaned, disinfected, rinsed with sterile water, and allowed to air-dry between uses. MDIs are designed for single-patient use; however, some laboratories have adopted a common canister protocol, which allows the MDI to be reused for multiple patients. ${ }^{12}$ This protocol requires that a clean valved holding chamber be used for each patient and that the MDI nozzle be cleaned and disinfected between patients. The canister itself is wiped with alcohol between patient uses, and good handwashing technique is required. The common canister protocol may not be appropriate for testing high-risk populations (eg, patients with cystic fibrosis, immunocompromised patients, lung transplant recipients).

Protocols for aerosolized bronchodilator delivery in conjunction with PFT underlie even larger problems of diagnosing and categorizing airway obstruction. These problems include how obstruction and reversibility of air-flow limitation are defined. ${ }^{13}$ The Global Initiative for Chronic 


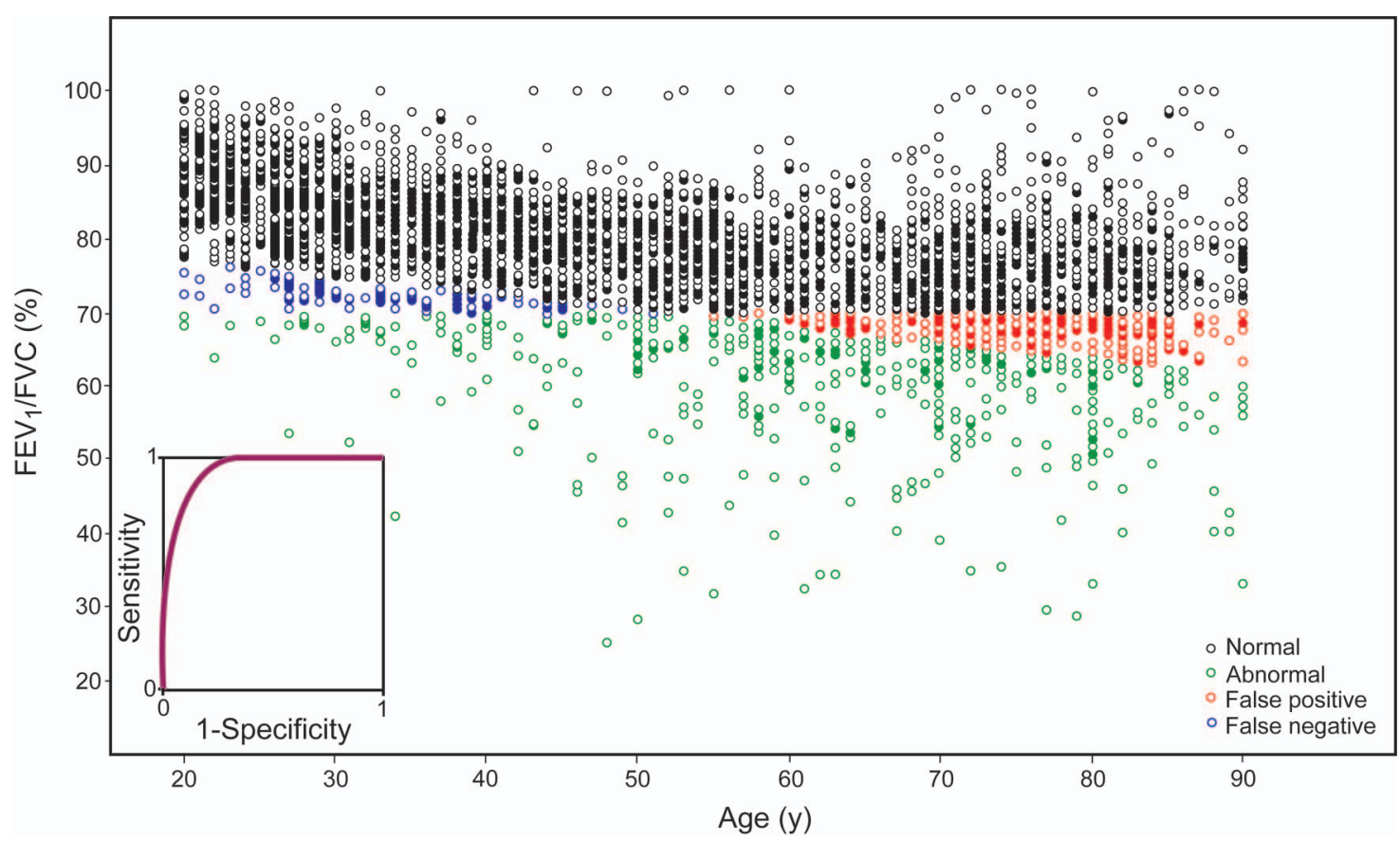

Fig. 1. $\mathrm{FEV}_{1} / \mathrm{FVC}$ ratios for females in the National Health and Nutrition Examination Survey III study are plotted against their age. The graph compares the fixed cutoff of $70 \%$ to the lower limit of the normal range (defined as the lowest percentile) for making a diagnosis of airway obstruction. Black circles represent subjects with normal values (true negative) by either method, and green circles represent subjects with abnormal ratios (true positive) by either method. Red circles show false positives, that is, subjects whose $\mathrm{FEV} / \mathrm{FVC}_{1}$ is $<0.70$ but above the lowest 5 th percentile. Blue circles represent younger subjects who are false negatives because their ratios are $>0.70$ but below the lowest 5th percentile. Using the fixed cutoff introduces an age-related bias and may misclassify older subjects as having airway obstruction or younger patients as normal when their lung function is significantly below their lowest 5 th percentile. The receiver operating characteristic curve is shown in the inset. Courtesy Philip H Quanjer.

Obstructive Lung Disease (GOLD) defines COPD as $\mathrm{FEV}_{1} / \mathrm{FVC}<0.70$ after bronchodilator administration. ${ }^{14}$ The GOLD criteria do not specify any particular methodology for bronchodilator administration. This definition, based on a fixed cutoff of 0.70 , has been widely adopted for clinical and research purposes. Unfortunately, the construct of a fixed cutoff obscures the natural history of lung function. Both $\mathrm{FEV}_{1}$ and FVC decrease with advancing age; $\mathrm{FEV}_{1}$ decreases slightly more rapidly that $\mathrm{FVC}$, and as a result, the ratio decreases with age. ${ }^{15}$ In men, $\mathrm{FEV}_{1}$ / FVC reaches 0.70 usually in the fourth to fifth decade of life, and in women, slightly later (fifth decade). Using a fixed cutoff after bronchodilator administration misclassifies older adults as having airway obstruction and may miss real obstruction in young adults (Fig. 1). Numerous studies, as well as the ATS/ERS guidelines, ${ }^{1}$ suggest using the lowest fifth percentile as the lower limit of the normal range to define airway obstruction. ${ }^{16-18}$

The second problem related to interpretation of postbronchodilator pulmonary function is how reversibility is defined. Current ATS/ERS recommendations suggest that a post-bronchodilator increase in $\mathrm{FEV}_{1}$ (or FVC) of $>12 \%$ and $0.2 \mathrm{~L}$ is the minimally significant change. ${ }^{19}$ Some clinicians prefer an improvement in the percent predicted following bronchodilator administration; in this case, an increase of $>9 \%$ may be considered significant. ${ }^{20}$ These definitions of reversibility are based on changes in $\mathrm{FEV}_{1}$ that exceed those occurring in healthy subjects following bronchodilator administration. Healthy subjects typically have small improvements (3-10\%). ${ }^{19}$ However, requiring a $12 \%$ and $0.2-\mathrm{L}$ increase may not be appropriate in older subjects with obstruction. The 12\%/0.2-L criteria are not appropriate for many pediatric subjects. ${ }^{21}$ Many patients with baseline airway obstruction show symptom relief even though they do not formally meet the ATS/ERS criteria. Subjects with COPD have shown quite variable responses to inhaled bronchodilators upon serial testing (Fig. 2). ${ }^{22}$

Another issue complicating the interpretation of postbronchodilator pulmonary function is whether other tests besides spirometry should be used. Lung-volume measurements are used infrequently but may show significant changes, particularly in the presence of hyperinflation or air trapping. ${ }^{23}$ Airway resistance and conductance, measured by body plethysmography, often improve significantly even though $\mathrm{FEV}_{1}$ does not. Because of the variability in airway resistance and conductance in healthy subjects, improvements of $40-50 \%$ are necessary to conclude that reversibility is present. ${ }^{24}$ Measurements of re- 


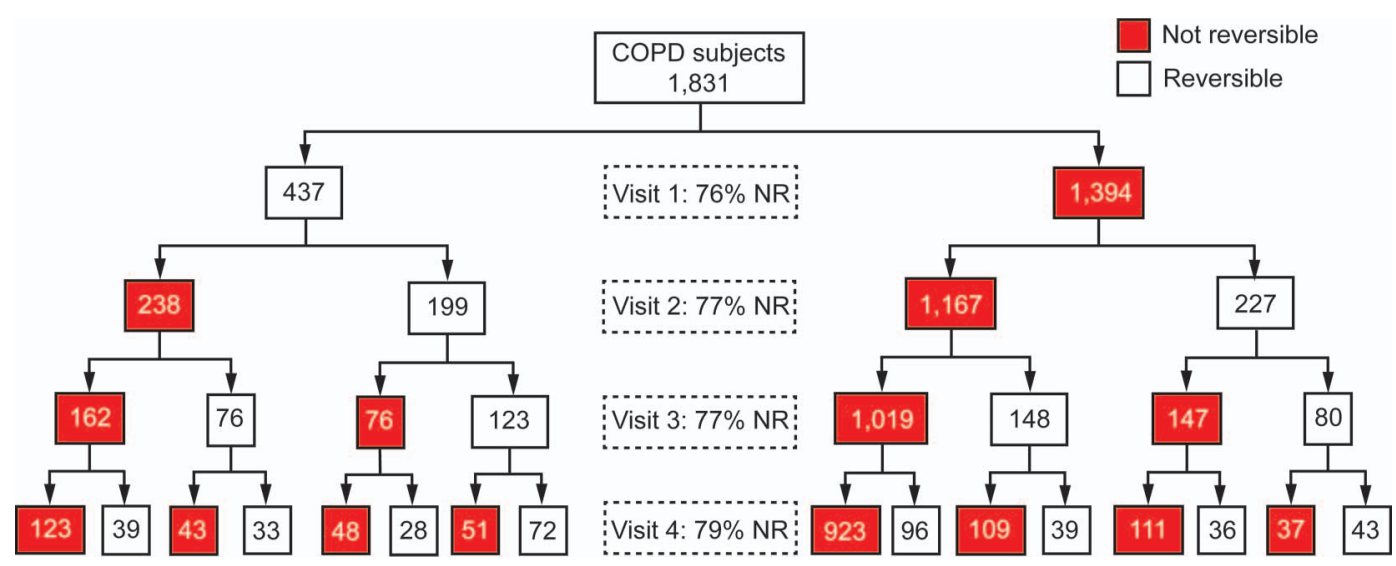

Fig. 2. Response to inhaled bronchodilator in 1,831 subjects with COPD on serial testing at 4 visits. Red boxes show the number of subjects who did not have a $12 \%$ and $0.2-\mathrm{L}$ improvement following inhaled bronchodilator administration. White boxes show the number of subjects who met the criteria for a positive response at each visit. Although between $76 \%$ and $79 \%$ of the subjects were reversible at each of the 4 visits, only $50 \%(923 / 1,831)$ were not reversible (NR) at all visits, and only $4 \%(72 / 1,831)$ displayed consistent reversibility at all visits. Data are from Reference 22.

Table 1. Bronchodilator Testing Problems

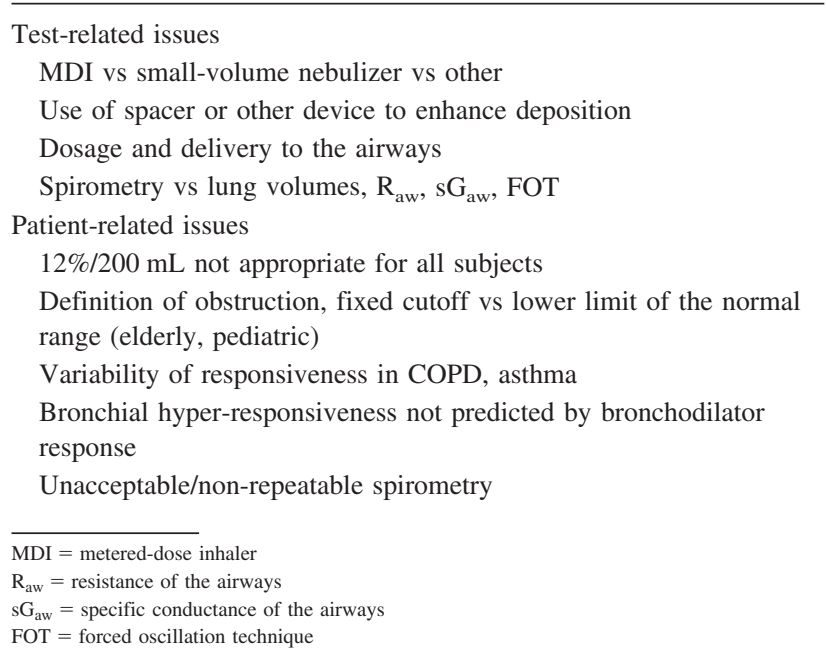

sistance by the forced oscillation technique may show similar improvements after bronchodilation, and this methodology is applicable to pediatric patients who may not be able to perform spirometry. ${ }^{25}$

Table 1 summarizes some of the problems surrounding pre/post-bronchodilator testing and interpretation of reversibility. Because of these, the consensus of most clinicians is that lack of response to a single measurement does not preclude a trial of bronchodilators. The consequences of false-positive and false-negative results must be considered. A false positive (apparent bronchodilator response when none is present) usually results in prescription of a bronchodilator, which may present risks, particularly in patients who have cardiovascular comorbidities. ${ }^{26} \mathrm{~A}$ false negative (no apparent response in the presence of airway obstruction) can result in misclassification and misdiagnosis. A false-negative response to bronchodilator testing can be particularly insidious in cases in which an elderly patient is misclassified as having airway obstruction. Such individuals may be diagnosed as having COPD. ${ }^{16}$ The following points should be considered in the performance and interpretation of bronchodilator studies: 1) reduce variability by using a consistent method of bronchodilator administration, 2) consider the rationale for testing (overall improvement vs reversibility), 3 ) recognize the variability of an individual patient's response (especially in COPD), 4) consider responses in addition to $\mathrm{FEV}_{1}$ (residual volume, specific airway conductance, forced oscillation technique), and 5) a negative response does NOT preclude a clinical trial.

\section{Inhaled Bronchial Challenge Agents}

Bronchial challenges are designed to answer 2 basic questions: 1) Does the patient have asthma? 2) What is the degree of hyper-responsiveness? Inhalation challenge tests are widely used to assist in making or excluding the diagnosis of asthma. ${ }^{4,27}$ Aerosols are used for both direct (methacholine, histamine) and indirect (mannitol, hypertonic saline) bronchial challenges. Table 2 lists the agents available for direct and indirect bronchial challenge. Direct challenge agents cause bronchoconstriction in the smooth muscle surrounding the airways, whereas indirect challenge agents provoke changes in the mucociliary lining of the airways, similar to those that accompany airway inflammation and narrowing. Direct challenge with methacholine has a high sensitivity and fair specificity if the challenge is performed close to the time when symptoms are present. Direct challenges are most useful to rule out 
Table 2. Direct and Indirect Bronchial Challenge Agents

\begin{tabular}{l}
\hline \hline Direct \\
Cholinergic agonists \\
Methacholine* \\
Carbachol* \\
Acetylcholine* \\
Histamine* \\
Indirect \\
Physical stimuli \\
Exercise \\
Eucapnic voluntary hyperventilation \\
Hypertonic saline* \\
Mannitol* \\
Pharmacologic stimuli \\
Adenosine monophosphate* \\
* Aerosol challenge agents \\
\hline
\end{tabular}

asthma, that is, a negative direct challenge effectively eliminates current asthma. Indirect challenges such as exercise or mannitol have a high specificity in patients who have airway inflammation common to asthma. A positive indirect challenge confirms (rules in) the presence of asthma and is often associated with exercise-induced bronchospasm. ${ }^{6}$ Unfortunately, inhalation challenges are subject to many of the same problems that plague bronchodilator studies, namely, the method of aerosol generation and delivery can greatly affect the outcome of the challenge. ${ }^{28}$ Additional issues that arise during inhalation challenges include the fact that the patient may experience symptoms and physiologic changes that make it difficult to perform the required maneuvers (maximum forced exhalation). ${ }^{29}$

Neither direct nor indirect challenges are 100\% sensitive and specific for asthma. Direct challenges (eg, methacholine) may result in a false positive in current smokers, patients with congestive heart failure or allergic rhinitis, and diseases that affect airway caliber (COPD, bronchitis, cystic fibrosis). Direct challenges can give a false negative if asthma-like symptoms are not present or if the patient has not withheld bronchodilators or other anti-asthma medications. Both direct and indirect challenges can result in a false positive if the patient has had a recent upper or lower respiratory tract infection. ${ }^{6}$ Indirect challenges (such as exercise or mannitol) may give a false negative if the patient has recently exercised (ie, indirect challenges tend to cause a refractory period) or if medications are not withheld appropriately. Paroxysmal coughing sometimes occurs with indirect challenges, making it difficult to assess the degree of bronchial hyper-responsiveness.

Hypertonic saline (and mannitol) may be used to promote airway clearance as well as for bronchial challenge. Hypertonic saline (3.0-7.0\%) has been used to assist with clearance of secretions in cystic fibrosis as well as in infants with bronchiolitis. A hypertonic saline challenge may

\begin{tabular}{|c|c|}
\hline \multicolumn{2}{|c|}{ Methacholine* Dosing Schemes } \\
\hline Doubling dose & Quadrupling dose \\
\hline $0.031 \mathrm{mg} / \mathrm{mL}$ & $0.0625 \mathrm{mg} / \mathrm{mL}$ \\
\hline $0.0625 \mathrm{mg} / \mathrm{mL}$ & $0.250 \mathrm{mg} / \mathrm{mL}$ \\
\hline $0.125 \mathrm{mg} / \mathrm{mL}$ & $\mathrm{mg} / \mathrm{mL}$ \\
\hline $0.250 \mathrm{mg} / \mathrm{mL}$ & $\mathrm{mg} / \mathrm{mL}$ \\
\hline $\mathrm{mg} / \mathrm{mL}$ & $\mathrm{mg} / \mathrm{mL}$ \\
\hline $\mathrm{mg} / \mathrm{mL}$ & \\
\hline $\mathrm{mg} / \mathrm{mL}$ & \\
\hline $\mathrm{mg} / \mathrm{mL}$ & \\
\hline $\mathrm{mg} / \mathrm{mL}$ & \\
\hline $\begin{array}{l}\mathrm{mg} / \mathrm{mL} \\
{ }^{*} \text { Also us }\end{array}$ & or histamine chall \\
\hline
\end{tabular}

Fig. 3. Two methacholine dosing schemes. Methacholine may be administered in 9 doubling doses from $0.031 \mathrm{mg} / \mathrm{mL}$ up to $16 \mathrm{mg} / \mathrm{mL}$ or using a quadrupling scheme with 5 doses ranging from $0.625 \mathrm{mg} / \mathrm{mL}$ up to $16 \mathrm{mg} / \mathrm{mL}$. A similar scheme can be used for histamine challenge. Not shown is an optional diluent step; normal saline or similar is administered before the first dose of methacholine and used to establish the target $\mathrm{FEV}_{1}$ for calculating a 20\% decrease. Data are from Reference 30.

be used to assess the safety of the agent for therapeutic purposes. In the standard challenge procedure, a high-output ultrasonic nebulizer or jet nebulizer is used to administer hypertonic saline $(4.5 \%)$ over progressively increasing intervals (eg, $30 \mathrm{~s}$ and 1, 2, 4, and $8 \mathrm{~min}$ ) with $\mathrm{FEV}_{1}$ measured after each dose. A decrease in $\mathrm{FEV}_{1}$ of $>15 \%$ is considered consistent with increased bronchial hyperreactivity. To evaluate patients with cystic fibrosis, a $\beta$ agonist is administered before the saline challenge. A decrease of $>20 \%$ or intolerable symptoms would cause the patient to be ineligible for hypertonic saline therapy.

In 2000, the ATS published guidelines for performance of direct challenges using methacholine. ${ }^{30}$ The recommendations suggest 2 protocols that can be used with the assumption that either will produce similar results when applied in a clinical setting evaluating subjects with suspected asthma. ${ }^{30}$ The suggested protocols are the 2-min tidal breathing and 5-breath dosimeter methods. Although these protocols differ significantly in their methodologies (see below), both can be interpreted by expressing their results in terms of the provocative concentration of methacholine that produces a $20 \%$ decrease in $\mathrm{FEV}_{1}\left(\mathrm{PC}_{20}\right)$.

For each of the protocols, 2 methacholine dosing schemes are suggested (Fig. 3). Each protocol starts with a very low concentration of methacholine $(0.031$ or $0.0625 \mathrm{mg} / \mathrm{mL})$ and increases with either doubling or quadrupling doses up to a maximum of $16 \mathrm{mg} / \mathrm{mL}$. Because the 9-dose doubling scheme takes significantly longer to administer, the 5-dose quadrupling scheme is used more frequently. It should be noted that other dosing schemes are sometimes used; in particular, the dosing scheme supplied with the commercially available brand of methacholine (Provocholine, Methapharm, Brantford, Ontario, Canada) uses 5 dilutions 


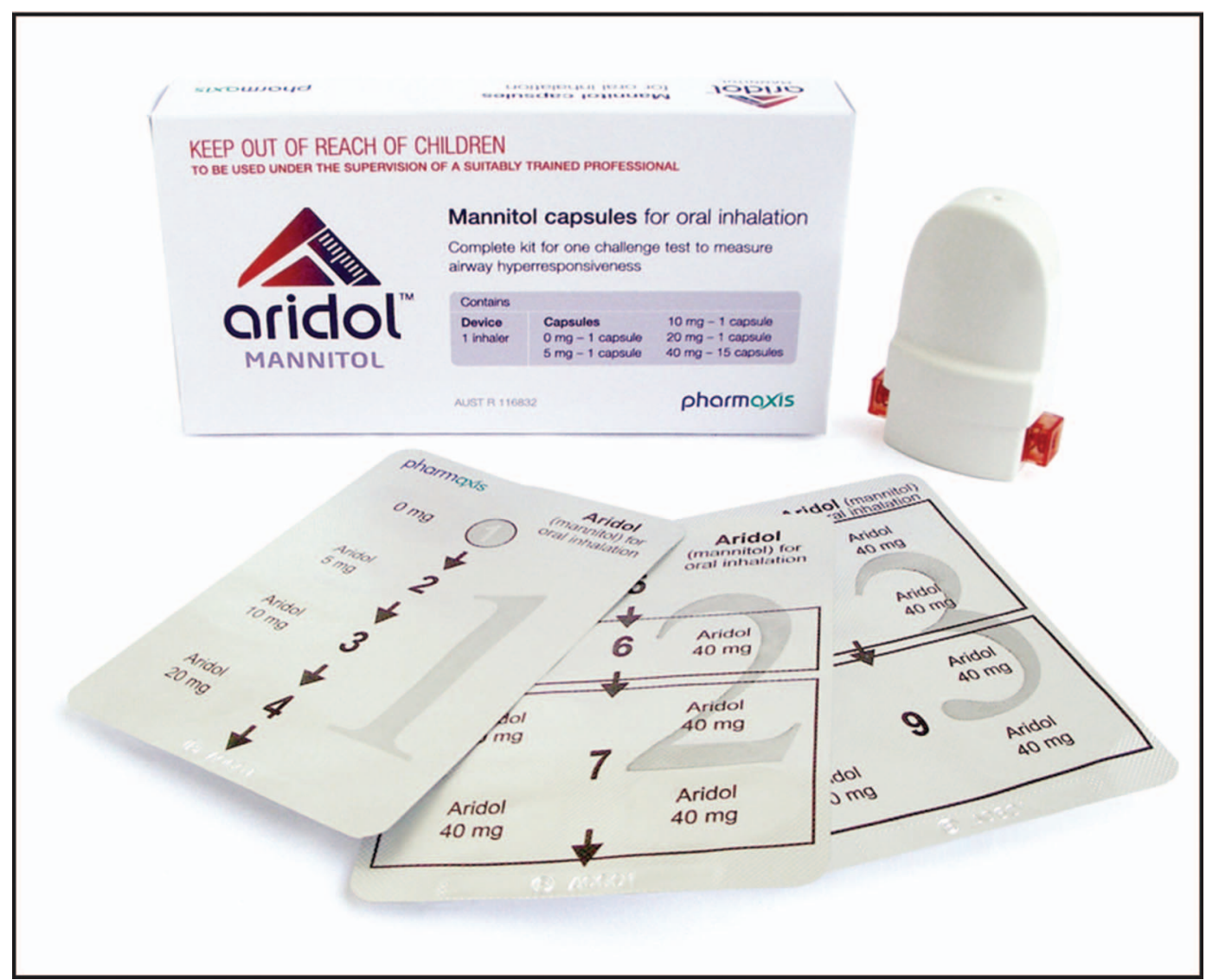

Fig. 4. Mannitol challenge kit, marketed as Aridol. Each kit contains numbered blister packs with capsules of mannitol powder along with a disposable proprietary powder inhaler. Courtesy Pharmaxis.

is simply the dose of drug corresponding to the $\geq 20 \%$ decrease in $\mathrm{FEV}_{1}$. If the patient has a $20 \%$ decrease in $\mathrm{FEV}_{1}$ following the first dose of methacholine (or after the diluent dose), $\mathrm{PC}_{20}$ cannot be calculated and should be reported as $<1 \mathrm{mg} / \mathrm{mL}$. If the patient does not have a $20 \%$ decrease after the highest dose has been administered, $\mathrm{PC}_{20}$ should be reported as $>16 \mathrm{mg} / \mathrm{mL}$. In addition to $\mathrm{FEV}_{1}$, tests such as specific airway conductance or forced oscillation may be useful in confirming an asthma diagnosis. ${ }^{34}$

Mannitol (Aridol in the United States) is a recent addition to the armamentarium of inhaled bronchial challenge agents. Mannitol is an indirect agent designed for rapid assessment of patients with asthma-like symptoms, particularly those related to exercise. ${ }^{35}$ Mannitol is a sugar alcohol and is supplied as a powder with a proprietary inhaler (Fig. 4). As the powder is inhaled, an osmotic gradient is established in the airway mucosa, stimulating release of the same mediators responsible for exercise-induced bronchospasm. Table 6 lists the doses used in the standard kit.

The patient inhales from the powder inhaler, with nose clip in place, from functional residual capacity to total lung capacity with a 5-s breath-hold. The first capsule contains no active drug and serves as the control. Dupli-
Table 6. Mannitol Dosing Regimen

\begin{tabular}{lrcl}
\hline \hline Dose No. & $\begin{array}{r}\text { Dose } \\
(\mathrm{mg})\end{array}$ & $\begin{array}{c}\text { Cumulative } \\
\text { Dose }(\mathrm{mg})\end{array}$ & Capsules/Dose \\
\hline 1 & 0 & 0 & 1 \\
2 & 5 & 5 & 1 \\
3 & 10 & 15 & 1 \\
4 & 20 & 35 & 1 \\
5 & 40 & 75 & 1 \\
6 & 80 & 155 & $2 \times 40 \mathrm{mg}$ \\
7 & 160 & 315 & $4 \times 40 \mathrm{mg}$ \\
8 & 160 & 475 & $4 \times 40 \mathrm{mg}$ \\
9 & 160 & 635 & $4 \times 40 \mathrm{mg}$ \\
\hline
\end{tabular}

cate measurements of $\mathrm{FEV}_{1}$ are made $60 \mathrm{~s}$ after the inhalation. The target $\mathrm{FEV}_{1}$ is a $15 \%$ decrease from the control value or a $10 \%$ decrease between doses. If neither target is reached, the patient continues to the next higher dose. For the last 4 doses, multiple capsules are used (see Table 6). The technologist administering the test needs to make sure each dose is administered rapidly to maintain the osmotic gradient created in the airway. 
Table 7. Interpretation of Mannitol Challenge

\begin{tabular}{ll}
\hline \hline \multicolumn{1}{c}{$\mathrm{PD}_{15}$} & \multicolumn{1}{c}{ Interpretation } \\
\hline$>635 \mathrm{mg} / \mathrm{mL}$ & Normal \\
$>155 \mathrm{mg} / \mathrm{mL}$ & Mild bronchial hyper-responsiveness \\
$\leq 155 \mathrm{mg} / \mathrm{mL}$ & Moderate bronchial hyper-responsiveness \\
$\leq 35 \mathrm{mg} / \mathrm{mL}$ & Severe bronchial hyper-responsiveness \\
& \\
$\mathrm{PD}_{15}=$ provocative dose of mannitol required to produce a 15\% decrease in $\mathrm{FEV}_{1}$ \\
\hline
\end{tabular}

If the patient has a $15 \%$ decrease in $\mathrm{FEV}_{1}$ or a $10 \%$ decrease between doses, the test is considered positive, and the cumulative dose is reported as the provocative dose. If the patient inhales all 9 doses (cumulative dose of $635 \mathrm{mg}$ ) without a $15 \%$ decrease in $\mathrm{FEV}_{1}$, the test is considered negative. The powder sometimes causes excessive coughing (with or without a significant decrease in $\mathrm{FEV}_{1}$ ), which also suggests airway hyper-responsiveness. Table 7 categorizes the response to inhaled mannitol by cumulative dose.

Mannitol and methacholine have similar sensitivity and specificity when used to evaluate subjects with symptoms of exercise-induced bronchospasm. ${ }^{36}$ However, there is significant variability in how individual patients respond to either drug, particularly if gauged against exercise response. Because mannitol is a physical indirect agent, it is recognized by the International Olympic Committee (along with exercise, eucapnic hyperventilation, and hypertonic saline) as a preferred means to determine airway hyperresponsiveness in elite athletes. ${ }^{37}$

\section{Safety During Bronchoprovocation}

Both methacholine and mannitol have similar safety profiles when used according to recommended protocols. Methacholine is considered safe and effective for patients $5 \mathrm{y}$ of age and older. It is contraindicated in women who are pregnant, who may become pregnant, or who are lactating. Methacholine produces asthma-like symptoms in susceptible patients. ${ }^{31}$ It is not recommended when the patient has a reduced $\mathrm{FEV}_{1}$, but has been shown to be safe even if $\mathrm{FEV}_{1}$ is low. ${ }^{38}$ Safe use of methacholine aerosol requires careful attention to the patient's symptoms as well as terminating the test when $\mathrm{FEV}_{1}$ decreases below a fixed threshold (ie, 20\%). ${ }^{30}$

Mannitol is safe and effective and may be used in patients $6 \mathrm{y}$ of age and older. It is not recommended for women who are pregnant or lactating; its use in these patients must be carefully considered, weighing the benefit of measuring airway hyper-responsiveness against the risk. ${ }^{39}$ Like methacholine, it is not recommended in patients whose $\mathrm{FEV}_{1}$ is already reduced $(<1.0-1.5 \mathrm{~L}$ or $<70 \%$ of predicted). Mannitol may cause significant cough and/or throat irritation in some individuals. Testing should be terminated when $\mathrm{FEV}_{1}$ decreases below a fixed threshold (ie, $15 \%$ or $10 \%$ between doses).

Aerosolized methacholine and mannitol are both designed to induce bronchospasm as a diagnostic test. Because there is a possibility of severe bronchospasm, these tests should be conducted by technologists who are familiar with the actions and side effects of the drugs and only under the supervision of a physician. ${ }^{30}$ Medications and equipment to treat severe bronchospasm must be immediately available in the testing area. Some patients may have comorbidities for which severe coughing or induced bronchospasm may be harmful. These include, but are not limited to, recent myocardial infarction, severe angina, uncontrolled hypertension, and upper or lower respiratory tract infections.

The efficacy of both methacholine and mannitol can be affected by the concomitant use of bronchodilators or other anti-asthma preparations. Patients who use inhaled $\beta$ agonists on a daily basis may have a reduced response to those drugs when used to reverse bronchospasm induced during bronchial challenge. ${ }^{40}$ The effects of $\beta$ blockers on bronchial hyper-reactivity is not well defined, but these agents may diminish the ability of $\beta$ agonists to reverse induced bronchospasm. ${ }^{41}$

The method of aerosol administration can also influence the measurement of bronchial hyper-responsiveness. Both the 5-breath dosimeter method (methacholine) and the standard mannitol protocol require the patient to inspire maximally from functional residual capacity to total lung capacity and perform a breath-hold. The effect of this type of deep inspiration is well documented. ${ }^{42}$ In normal patients, deep inspiration causes mild bronchodilation, and this may also be seen in patients with mild asthma. In some patients who have marked airway hyper-responsiveness, deep inspiration may have little or no effect, instead of having a bronchoprotective effect. The result of deep inspiration during the administration of the bronchial challenge aerosol is to attenuate the resulting bronchoconstriction. For methacholine challenge, there may be as much as a 2-fold difference in $\mathrm{PC}_{20}$, depending on whether a deep inspiration or tidal breathing technique is used. ${ }^{43}$ The effect of deep inspiration must be accounted for when selecting a bronchial challenge protocol and when interpreting the results.

\section{Summary}

Aerosolized bronchodilators are widely used to assess reversibility of airway obstruction and, in many instances, to establish a diagnosis of either COPD or asthma. In spite of their ubiquitous use, neither the equipment nor protocols are widely standardized. This lack of standardization is largely caused by the number of different inhaled bronchodilator medications and their varied delivery mecha- 
nisms. Even when standardized within an individual PFT lab, the response to inhaled bronchodilators is markedly variable, especially in patients with COPD.

Aerosolized bronchial challenge agents are similarly widely used. Protocols for methacholine challenge and, more recently, mannitol challenge are standardized. However, bronchial challenges using inhaled aerosols are subject to many of the same variables that are problematic in bronchodilator testing (method of nebulization, patient performance). Bronchial challenge with inhaled agents poses little risk to the patient when the prescribed protocols are followed because testing can be terminated at a predetermined threshold (unlike exercise-induced asthma or eucapnic voluntary hyperventilation tests). The method of aerosol administration can significantly affect the measured hyper-responsiveness, especially in protocols than involve deep inspiration.

\section{REFERENCES}

1. Miller MR, Hankinson J, Brusasco V, Burgos F, Casaburi R, Coates A, et al. Standardisation of spirometry. Eur Respir J 2005;26(2):319-338.

2. Coates AL, Graham BL, McFadden RG, McParland C, Moosa D, Provencher S, et al. Spirometry in primary care. Can Respir J 2013; 20(1):13-21.

3. Vestbo J, Hurd SS, Agustí AG, Jones PW, Vogelmeier C, Anzueto A, et al. Global strategy for the diagnosis, management, and prevention of chronic obstructive pulmonary disease: GOLD executive summary. Am J Respir Crit Care Med 2013;187(4):347-365.

4. Cockcroft DW, Davis BE. Diagnostic and therapeutic value of airway challenges in asthma. Curr Allergy Asthma Rep 2009:9(3):247-253.

5. Wanger J. ATS pulmonary function laboratory management and procedure manual. New York: American Thoracic Society; 2004; Chapter 7.

6. Cockcroft DW. Direct challenge tests-airway hyperresponsiveness in asthma: its measurement and clinical significance. Chest 2010; 138(2 Suppl):18S-24S.

7. Anderson SD. Indirect challenge tests-airway hyperresponsiveness in asthma: its measurement and clinical significance. Chest 2010; 138(2 Suppl):25S-30S.

8. Smith KJ, Chan HK, Brown KF. Influence of flow rate on aerosol particle size distributions from pressurized and breath-actuated inhalers. J Aerosol Med 1998;11(4):231-245.

9. Tal A, Golan H, Grauer N, Aviram M, Albin D, Quastel MR. Deposition pattern of radiolabeled salbutamol inhaled from a meterdose inhaler by means of a spacer with mask in young children with airway obstruction. J Pediatr 1996;128(4):479-484.

10. Rottier BL, Rubin BK. Asthma medication delivery: mists and myths. Paediatr Respir Rev 2013;14(2):112-118.

11. O'Malley CA. Device cleaning and infection control in aerosol therapy. Respir Care 2015;60(6):••••.

12. Sheils SG, Duncan JL, Wojciechowski WV. The common canister protocol using the Monaghan AeroChamber reveals no cross-contamination and potential cost savings. Respir Care 2000;45(8):981.

13. Calverley PM, Albert P, Walker PP. Bronchodilator reversibility in chronic obstructive pulmonary disease: use and limitations. Lancet Respir Med 2013;1(7):564-573.

14. Global Initiative for Chronic Obstructive Lung Disease (GOLD). Global strategy for diagnosis, management, and prevention of COPD. http://www.goldcopd.org/Guidelines/guidelines-resources.html. Accessed April 21, 2014.
15. Swanney MP, Ruppel G, Enright PL, Pedersen OF, Crapo RO, Miller $\mathrm{MR}$, et al. Using the lower limit of normal for the $\mathrm{FEV}_{1} / \mathrm{FVC}$ ratio reduces the misclassification of airway obstruction. Thorax 2008; 63(12):1046-1051.

16. Roberts SD, Farber MO, Knox KS, Phillips GS, Bhatt NY, Mastronarde JG, Wood KL. FEV $/$ FVC ratio of $70 \%$ misclassifies patients with obstruction at the extremes of age. Chest 2006;130(1):200-206.

17. Aggarwal AN, Gupta D, Behera D, Jindal SK. Comparison of fixed percentage method and lower confidence limits for defining limits of normality for interpretation of spirometry. Respir Care 2006;51(7): 737-743.

18. Hansen JE, Sun XG, Wasserman K. Spirometric criteria for airway obstruction. Use percentage of $\mathrm{FEV}_{1} / \mathrm{FVC}$ ratio below the fifth percentile, not $<70 \%$. Chest 2007;131(2):349-355.

19. Pellegrino R, Viegi G, Brusasco V, Crapo RO, Burgos F, Casaburi $\mathrm{R}$, et al. Interpretative strategies for lung function tests. Eur Respir $\mathrm{J}$ 2005;26(5):948-968.

20. Bussamra MH, Cukier A, Stelmach R, Rodrigues JC. Evaluation of the magnitude of the bronchodilator response in children and adolescents with asthma. Chest 2005;127(2):530-535.

21. Seed L, Wilson D, Coates AL. Children should not be treated like little adults in the PFT lab. Respir Care 2012;57(1):61-70; discussion 71-74.

22. Albert P, Agusti A, Edwards L, Tal-Singer R, Yates J, Bakke P, et al. Bronchodilator responsiveness as a phenotypic characteristic of established chronic obstructive pulmonary disease. Thorax 2012; 67(8):701-708.

23. Deesomchok A, Webb KA, Forkert L, Lam YM, Ofir D, Jensen D, O'Donnell DE. Lung hyperinflation and its reversibility in patients with airway obstruction of varying severity. COPD 2010;7(6):428-437.

24. Borrill ZL, Houghton CM, Tal-Singer R, Vessey SR, Faiferman I, Langley SJ, Singh D. The use of plethysmography and oscillometry to compare long-acting bronchodilators in patients with COPD. Br J Clin Pharmacol 2007;65(2):244-252.

25. Nielsen KG, Bisgaard H. Discriminative capacity of bronchodilator response measured with three different lung function techniques in asthmatic and healthy children aged 2 to 5 years. Am J Respir Crit Care Med 2001;164(4):554-559.

26. Wise RA, Anzueto A, Cotton D, Dahl R, Devins T, Disse B, et al. Tiotropium Respimat inhaler and the risk of death in COPD. N Engl J Med 2013;369(16):1491-1501.

27. Anderson SD. Provocative challenges to help diagnose and monitor asthma: exercise, methacholine, adenosine, and mannitol. Curr Opin Pulm Med 2008;14(1):39-45.

28. Casset A, Purohit A, Birba E, Chenard MP, Uring Lambert B, Bahram $S$, et al. Bronchial challenge test in asthmatics sensitized to mites: role of particle size in bronchial response. J Aerosol Med 2007;20(4):509-518.

29. Pedrosa M, Barranco P, Caminoa M, Botello MD, Quirce S. Comparison of methacholine and adenosine inhalation challenge in patients with suspected asthma. J Asthma 2009;46(8):773-776.

30. Crapo RO, Casaburi R, Coates AL, Enright PL, Hankinson JL, Irvin CG, et al. American Thoracic Society: guidelines for methacholine and exercise challenge testing-1999. Am J Respir Crit Care Med 2000;161(1):309-329.

31. Dilution sequence-single patient testing. Provocholine package insert. Brantford, Ontario, Canada: Methapharm; 2004.

32. Ryan G, Dolovich MB, Obminski G, Cockcroft DW, Juniper E, Hargreave FE, Newhouse MT. Standardization of inhalation provocation tests: influence of nebulizer output, particle size, and method of inhalation. J Allergy Clin Immunol 1981;67(2):156-161.

33. Shapiro GG, Simon RA. Bronchoprovocation Committee. Bronchoprovocation committee report. American Academy of Allergy and Immunology. J Allergy Clin Immunol 1992;89(3):775-778. 
34. Gartman EJ, Dinino EK, Koo P, Roberts MB, McCool FD. Methacholine-induced airway hyper-reactivity phenotypes. J Asthma 2013; 50(6):629-633.

35. Brannan JD, Koskela H, Anderson SD, Chew N. Responsiveness to mannitol in asthmatic subjects with exercise- and hyperventilation-induced asthma. Am J Respir Crit Care Med 1998;158(4):1120-1126.

36. Anderson SD, Charlton B, Weiler JM, Nichols S, Spector SL, Pearlman DS, A305 Study Group. Comparison of mannitol and methacholine to predict exercise-induced bronchoconstriction and a clinical diagnosis of asthma. Respir Res 2009;10(1):4-17.

37. Fitch KD, Sue-Chu M, Anderson SD, Boulet LP, Hancox RJ, McKenzie DC, et al. Asthma and the elite athlete: summary of the International Olympic Committee's consensus conference, Lausanne, Switzerland, January 22-24, 2008. J Allergy Clin Immunol 2008;122(2):254.e7260.e7.

38. Martin RJ, Wanger JS, Irvin CG, Bucher Bartelson B, Cherniack RM. Methacholine challenge testing: safety of low starting $\mathrm{FEV}_{1}$.
Asthma Clinical Research Network (ACRN). Chest 1997;112(1): 53-56.

39. Aridol (mannitol inhalation powder) bronchial challenge test kit. Package insert for Aridol. Exton, PA: Pharmaxis; 2010.

40. Haney S, Hancox RJ. Rapid onset of tolerance to beta-agonist bronchodilation. Respir Med 2005;99(5):566-571.

41. Short PM, Williamson PA, Anderson WJ, Lipworth BJ. Randomized placebo-controlled trial to evaluate chronic dosing effects of propranolol in asthma. Am J Respir Crit Care Med 2013;187(12): 1308-1314.

42. Pellegrino R, Sterk PJ, Sont JK, Brusasco V. Assessing the effect of deep inhalation on airway calibre: a novel approach to lung function in bronchial asthma and COPD. Eur Respir J 1998;12(5):1219-1227.

43. Allen ND, Davis BE, Hurst TS, Cockcroft DW. Difference between dosimeter and tidal breathing methacholine challenge-contributions of dose and deep inspiration bronchoprotection. Chest 2005;128(6): 4018-4023.

\section{Discussion}

Berlinski: Thank you for that nice presentation. Based on what you said, it would be fair to say that the tidal breathing method would be the most appropriate method for our patients In addition, you have flow-dependent dosing with DPI [dry powder inhaler] If you do a deep inhalation, you have a bronchoprotective effect. The 2-min tidal breathing method could be used with younger children to adults.

Ruppel: Yes, I think you're going to see less use of the dosimeter because even the modified dosimeter method is going to be somewhat problematic. But there are a lot of dosimeters around. I didn't mention it, but the folks here below $2 \mathrm{mg} / \mathrm{mL}$, they're pretty consistent, so if there's significant airway hyper-responsiveness, the method doesn't make quite as much difference. But because the dosimeter delivers about half as much total drug by certain estimations, that probably does play a role somewhere in the middle here. These folks here would probably be protected by deep inspiration, but the good news is that if you can get the drug down in the airways and we continue using $\mathrm{PC}_{20}$ [provocative concentration that produces a $20 \%$ decrease in $\mathrm{FEV}_{1}$ ], not the cumulative breath units or some of the other things we used in the past, you're probably going to be able to detect and answer the first question: does the patient have asthma? Whether you' 11 be able to classify the responsiveness of the patient is another story.

\$ Suggett: I've got a doubt about the DPI use of mannitol. You touched on flow dependence, and it was also mentioned this morning in terms of adherence and using the right methodology. For this type of device (DPI), in which you have to disperse the powder to get a good fine-particle fraction, but you also have the potential of flow dependence influencing the degree of powder dispersion, are there any data to show consistency of dosing across different patients in reality?

Ruppel: I believe there is some literature from Sandy Anderson's group,${ }^{1}$ who looked at that in deposition studies. They saw that it did work and was well controlled, but there was pretty good deposition in the lower airways as well as in the larger airways, with some variability in the penetration index that was not flow-dependent. The biggest issue we've seen from a practical view point is if the patient inhales too rapidly, the little capsule spins too fast, static charge builds up within the device, which makes it hard to get the capsule out, some of the powder adheres to the filter in the device, and a lot of the drug impacts in the upper airway, and the patient begins to cough. The trick is to get the patient to inhale rapidly enough to cause the spinning action but not so fast that all those other things happen; it's really kind of an art that the technologist who's using it has to carefully teach the patient. The Aridol folks give you a little practice device to use, but you know how patients are: they're going to do what they're going to do, and when you tell them to take a big breath, they'll suck in really hard, and you're off to the cough.

\$ Suggett: It looks like it is a conventional capsule-based DPI, and I am therefore pretty sure there will be variability with that.

Ruppel: Yes. We were more concerned with the static charge that was building up in there and getting the capsule out, so we actually tried having technologists put their hands on an antistatic mat that you'd use if you were working on sensitive electronics, and it didn't help at all. The best thing is to wear gloves and have tweezers to get the capsule out, but it's very technique-dependent.

$\ddagger$ Jason A Suggett PhD MBA, Monaghan Medical.

\section{REFERENCES}

1. Glover W, Chan HK, Eberl S, Daviskas E, Anderson S. Lung deposition of mannitol powder aerosol in healthy subjects. J Aerosol Med 2006;19(4):522-532. 